\title{
The functional inequality for the mixed quermassintegral
}

\author{
Fangwei Chen ${ }^{1}$, Jianbo Fang ${ }^{1}$, Miao Luo ${ }^{2}$ and Congli Yang ${ }^{2 *}$ (D)
}

\author{
"Correspondence: \\ yangcongli@gznu.edu.cn \\ ${ }^{2}$ School of Mathematical Sciences, \\ Guizhou Normal University, \\ Guiyang, Guizhou, China \\ Full list of author information is \\ available at the end of the article
}

\begin{abstract}
In this paper, the functional Quermassintegrals of a log-concave function in $\mathbb{R}^{n}$ are discussed. The functional inequality for the ith mixed Quermassintegral is established. Moreover, as a special case, a weaker log-Quermassintegral inequality in $\mathbb{R}^{n}$ is obtained.
\end{abstract}

Keywords: Log-concave function; Quermassintegral; Mixed Quermassintegral inequality; Minkowski inequality

\section{Introduction}

Let $\mathcal{K}^{n}$ be the set of convex bodies (compact convex subsets with nonempty interiors) in $\mathbb{R}^{n}$, the fundamental Brunn-Minkowski inequality for convex bodies states that, for $K, L \in$ $\mathcal{K}^{n}$, the volume of the bodies and of their Minkowski sum $K+L=\{x+y: x \in K$, and $y \in L\}$ are given by

$$
V(K+L)^{\frac{1}{n}} \geq V(K)^{\frac{1}{n}}+V(L)^{\frac{1}{n}}
$$

with equality if and only if $K$ and $L$ are homothetic, namely agreeing up to a translation and a dilation. The Brunn-Minkowski inequality exposes the crucial logarithmic concavity of the volume in $\mathcal{K}^{n}$, because it has an equivalent formulation as

$$
V((1-t) K+t L) \geq V(K)^{1-t} V(L)^{t}
$$

for $t \in(0,1)$. See for example $[18,19,29]$ for more about the Brunn-Minkowski inequality. Another important geometric inequality related to the convex bodies $K$ and $L$ is the mixed Quermassintegral inequality,

$$
W_{i}(K, L)^{n-i} \geq W_{i}(K)^{n-i-1} W_{i}(L), \quad 0 \leq i<n-1,
$$

with equality if and only if $K$ and $L$ are homothetic. Here $W_{i}(K)(i=0,1, \ldots, n)$ are the Quermassintegrals of $K$, which are defined by letting $W_{0}(K)=V_{n}(K)$, the volume of $K$;

c) The Author(s) 2020. This article is licensed under a Creative Commons Attribution 4.0 International License, which permits use, sharing, adaptation, distribution and reproduction in any medium or format, as long as you give appropriate credit to the original author(s) and the source, provide a link to the Creative Commons licence, and indicate if changes were made. The images or other third party material in this article are included in the article's Creative Commons licence, unless indicated otherwise in a credit line to the material. If material is not included in the article's Creative Commons licence and your intended use is not permitted by statutory regulation or exceeds the permitted use, you will need to obtain permission directly from the copyright holder. To view a copy of this licence, visit http://creativecommons.org/licenses/by/4.0/. 
$W_{n}(K)=\omega_{n}$, the volume of the unit ball $B_{2}^{n}$ in $\mathbb{R}^{n}$ and, for general $i=1,2, \ldots, n-1$,

$$
W_{n-i}(K)=\frac{\omega_{n}}{\omega_{i}} \int_{\mathcal{G}_{i, n}} \operatorname{vol}_{i}\left(\left.K\right|_{\xi_{i}}\right) d \mu\left(\xi_{i}\right)
$$

where the $\mathcal{G}_{i, n}$ is the Grassmannian manifold of $i$-dimensional linear subspaces of $\mathbb{R}^{n}$, $d \mu\left(\xi_{i}\right)$ is the normalized Haar measure on $\mathcal{G}_{i, n},\left.K\right|_{\xi_{i}}$ denotes the orthogonal projection of $K$ onto the $i$-dimensional subspaces $\xi_{i}$, and $\operatorname{vol}_{i}$ is the $i$-dimensional volume on space $\xi_{i}$.

In the 1960s, the Minkowski addition was extended to the $L^{p}(p \geq 1)$ Minkowski sum by defining $h_{K+p t \cdot L}^{p}=h_{K}^{p}+t h_{L}^{p}$. The extension of the mixed Quermassintegrals to the $L^{p}$ mixed Quermassintegrals is due to Lutwak [24]. The inequalities for the $L^{p}$ mixed Quermassintegrals are established by Lutwak. Let $K, L \in \mathcal{K}^{n}$ with origin in their interiors, $0 \leq i<n-i$ and $p>1$, then

$$
W_{p, i}(K, L)^{n-i} \geq W_{i}(K)^{n-i-p} W_{i}(L)^{p},
$$

with equality if and only if $K$ and $L$ are dilates. Here the $L^{p}$ mixed Quermassintegrals are defined by

$$
W_{p, i}(K, L):=\frac{p}{n-i} \lim _{t \rightarrow 0^{+}} \frac{W_{i}\left(K+{ }_{p} t \cdot L\right)-W_{i}(L)}{t},
$$

for $i=0,1, \ldots, n-1$. In particular, for $p=1$ in $(0.4)$, it becomes $W_{i}(K, L)$, and $W_{p, 0}(K, L)$ is denoted by $V_{p}(K, L)$, which is called the $L_{p}$ mixed volume of $K$ and $L$.

Motivated by the analogy properties between the log-concave functions and the volume of convex bodies in $\mathcal{K}^{n}$, the interest in studying the log-concave functions has been considerably increasing. For example, the functional Blaschke-Santaló inequality for even logconcave function is discussed by Ball in [6, 7]; for the general case see [8, 17, 21, 28]. The mean width for a log-concave function is introduced by Klartag, Milman and Rotem (see $[22,26,27])$. The affine isoperimetric inequality for the log-concave function is proved by Artstein-Avidan, Klartag, Schütt and Werner [5]. The John ellipsoid for log-concave function has been establish by Gutiérrez, Merino Jiménez and Villa [2], the LYZ ellipsoid for log-concave function is established by Fang and Zhou [16]. See [1, 4, 9, 12-14, 23] for more about the pertinent results.

Let $f=e^{-u}, g=e^{-v}$ be log-concave functions, $\alpha, \beta>0$, the "sum" and "scalar multiplication" of log-concave functions are defined as

$$
\alpha \cdot f \oplus \beta \cdot g:=e^{-w}, \quad \text { where } w^{*}=\alpha u^{*}+\beta v^{*},
$$

here $w^{*}$ denotes as usual the Fenchel conjugate. The total mass integral $J(f)$ of $f$ is defined as $J(f)=\int_{\mathbb{R}^{n}} f(x) d x$. In [15], the quantity $\delta J(f, g)$, which is called the first variation of $J$ at $f$ along $g$, is defined by Colesanti and Fragalà,

$$
\delta J(f, g)=\lim _{t \rightarrow 0^{+}} \frac{J(f \oplus t \cdot g)-J(f)}{t} .
$$

The authors show that the functional form of Minkowski's first inequality is

$$
\delta J(f, g) \geq J(f)[\log J(g)+n]+\operatorname{Ent}(f),
$$


where $\operatorname{Ent}(f)$ is the entropy of $f \operatorname{defined}$ by $\operatorname{Ent}(f)=\int_{\mathbb{R}^{n}} f \log f d x-J(f) \log J(f)$. We have inequality in (0.5) if and only if there exist $x_{0} \in \mathbb{R}^{n}$ such that $g(x)=f\left(x-x_{0}\right)$.

Inspired by Ref. [15] of Colesanti and Fragalà, in this paper, we define the $i$ th functional Quermassintegrals $W_{i}(f)$ as the $i$-dimensional average total mass of $f$,

$$
W_{i}(f):=\frac{\omega_{n}}{\omega_{n-i}} \int_{\mathcal{G}_{n-i, n}} J_{n-i}(f) d \mu\left(\xi_{n-i}\right)
$$

where $J_{i}(f)$ denotes the $i$-dimensional total mass of $f$ defined in (3.1), $\mathcal{G}_{i, n}$ is the Grassmannian manifold of $\mathbb{R}^{n}$ and $d \mu\left(\xi_{n-i}\right)$ is the normalized measure on $\mathcal{G}_{i, n}$. The first variation of $W_{i}$ at $f$ along $g$ is defined by (see Definition 3.3)

$$
W_{i}(f, g)=\lim _{t \rightarrow 0^{+}} \frac{W_{i}(f \oplus t \cdot g)-W_{i}(f)}{t} .
$$

$W_{i}(f, g)$ is a natural extension of the mixed Quermassintegrals of convex bodies in $\mathbb{R}^{n}$, we call it the $i$ th functional mixed Quermassintegral. In fact, if one takes $f=\chi_{K}$, and $\operatorname{dom}(f)=$ $K \in \mathbb{R}^{n}$, then $W_{i}\left(\chi_{K}\right)$ turns out to be $W_{i}(K)$, and $W_{i}\left(\chi_{K}, \chi_{L}\right)$ equals $W_{i}(K, L)$. In this paper, our main result is to show the inequality for the $i$ th functional mixed Quermassintegrals. Let $\mathcal{A}^{\prime}$ denote the integrable functions in $\mathbb{R}^{n}$.

Theorem 0.1 Let $f$ and $g$ are integrable functions on $\mathcal{A}^{\prime}$, then

$$
W_{i}(f, g) \geq W_{i}(f)\left[1+\frac{1}{n-i} \log \frac{W_{i}(g)}{W_{i}(f)}\right]+\frac{1}{n-i} W_{i}(f \log f)
$$

with equality if and only if there exists $x_{0} \in \mathbb{R}^{n}$ such that $g(x)=f\left(x-x_{0}\right)$, for all $x \in \mathbb{R}^{n}$.

The paper is organized as follows, in Sect. 1, we introduce some notations about the log-concave function. In Sect. 2, the projection of log-concave function is discussed. In Sect. 3, we turn our attention to the functional inequalities involving $W_{i}(f, g)$, we prove the $i$ th functional mixed Quermassintegral inequality. Specially, the weaker logQuermassintegral inequality for convex bodies is obtained as a corollary.

\section{Preliminaries}

Let $u: \Omega \rightarrow(-\infty,+\infty]$ be a convex function, that is, $u((1-t) x+t y) \leq(1-t) u(x)+t u(y)$ for $t \in(0,1)$, here $\Omega=\left\{x \in \mathbb{R}^{n}: u(x) \in \mathbb{R}\right\}$ is the domain of $u$. The convexity of $u$ implies that $\Omega$ is a convex set in $\mathbb{R}^{n}$. We say that $u$ is proper if $\Omega \neq \emptyset$, and $u$ is of class $\mathcal{C}_{+}^{2}$ if it is twice differentiable on $\operatorname{int}(\Omega)$, with a positive definite Hessian matrix. Let

$$
\begin{aligned}
\mathcal{L}= & \{u: \Omega \rightarrow(-\infty,+\infty]: \mathrm{u} \text { is convex, low semi-continuous } \\
& \text { and } \left.\lim _{\|x\| \rightarrow+\infty} u(x)=+\infty\right\} .
\end{aligned}
$$

The Fenchel conjugate of $u \in \mathcal{L}$ is defined by

$$
u^{*}(y)=\sup _{x \in \Omega}\{\langle x, y\rangle-u(x)\}
$$


It is obvious that $u(x)+u^{*}(y) \geq\langle x, y\rangle$ for all $x, y \in \Omega$, and there is an equality if and only if $x \in \Omega$, and $y$ is the subdifferential of $u$ at $x$, which means

$$
u^{*}(\nabla u(x))+u(x)=\langle x, \nabla u(x)\rangle
$$

Moreover, if $u$ is a lower semi-continuous convex function, then also $u^{*}$ is a lower semicontinuous convex function, and $u^{* *}=u$. The infimal convolution of functions $u$ and $v$ is defined by

$$
u \square v(x)=\inf _{y \in \Omega}\{u(x-y)+v(y)\} .
$$

The right scalar multiplication by a nonnegative real number $\alpha$ is given by

$$
(u \alpha)(x):= \begin{cases}\alpha u\left(\frac{x}{\alpha}\right) & \text { if } \alpha>0 \\ I_{\{0\}} & \text { if } \alpha=0\end{cases}
$$

The following propositions below gather some elementary properties of the Fenchel conjugate and the infimal convolution of $u$ and $v$, which can be found in [15, 25].

Proposition 1.1 Let $u \in \mathcal{L}$, then there exist constants $a$ and $b$, with $a>0$, such that for $x \in \Omega$

$$
u(x) \geq a\|x\|+b .
$$

Moreover, $u^{*}$ is proper and satisfies $u^{*}(y)>-\infty, \forall y \in \Omega$.

Proposition 1.2 Let $u, v: \Omega \rightarrow(-\infty,+\infty]$ are convex functions. Then:

1. $(u \square v)^{*}=u^{*}+v^{*}$;

2. $(u \alpha)^{*}=\alpha u^{*}, \alpha>0$;

3. $\operatorname{dom}(u \square v)=\operatorname{dom}(u)+\operatorname{dom}(v)$;

4. we have $u^{*}(0)=-\inf (u)$, in particular if $u$ is proper, then $u^{*}(y)>-\infty ; \inf (u)>-\infty$ implies $u^{*}$ is proper.

Proposition 1.3 Let $u: \Omega \rightarrow(-\infty,+\infty]$ be a closed convex function, and set $\mathcal{C}:=\operatorname{int}(\Omega)$, $\mathcal{C}^{*}:=\operatorname{int}\left(\operatorname{dom}\left(u^{*}\right)\right)$. Then $(\mathcal{C}, u)$ is a convex function of Legendre type if and only if $\left(C^{*}, u^{*}\right)$ is. In this case $\left(\mathcal{C}^{*}, u^{*}\right)$ is the Legendre conjugate of $(\mathcal{C}, u)$ (and conversely). Moreover, $\nabla u:=$ $\mathcal{C} \rightarrow \mathcal{C}^{*}$ is a continuous bijection, and the inverse map of $\nabla u$ is precisely $\nabla u^{*}$.

A function $f: \mathbb{R}^{n} \rightarrow(-\infty,+\infty]$ is called log-concave if, for $x, y \in \mathbb{R}^{n}$ and $0<t<1$, we have $f((1-t) x+t y) \geq f^{1-t}(x) f^{t}(y)$. If $f$ is a strictly positive log-concave function on $\mathbb{R}^{n}$, then there exists a convex function $u: \Omega \rightarrow(-\infty,+\infty]$ such that $f=e^{-u}$. The log-concave function is closely related to the convex geometry of $\mathbb{R}^{n}$. An example of a log-concave function is the characteristic function $\chi_{K}$ of a convex body $K$ in $\mathbb{R}^{n}$, which is defined by

$$
\chi_{K}(x)=e^{-I_{K}(x)}= \begin{cases}1 & \text { if } x \in K \\ 0 & \text { if } x \notin K,\end{cases}
$$


where $I_{K}$ is a lower semi-continuous convex function, and the indicator function of $K$ is

$$
I_{K}(x)= \begin{cases}0 & \text { if } x \in K \\ \infty & \text { if } x \notin K\end{cases}
$$

Let us generalize $f$ to the domain of $\mathbb{R}^{n}$ by

$$
\bar{f}= \begin{cases}f, & x \in \Omega ; \\ 0, & x \in \mathbb{R}^{n} / \Omega .\end{cases}
$$

In the later sections, we also use $f$ to denote $f$ having been extended to $\mathbb{R}^{n}$, let $\mathcal{A}=\{f$ : $\left.\mathbb{R}^{n} \rightarrow(0,+\infty]: f=e^{-u}, u \in \mathcal{L}\right\}$ be the subclass of $f$.

Definition 1.1 Let $f, g \in \mathcal{A}$, and $\alpha, \beta \geq 0$. The sum and multiplication of $f$ and $g$ are defined by $\alpha \cdot f \oplus \beta \cdot g=e^{-[(u \alpha) \square(v \beta)]}$. That means

$$
(\alpha \cdot f \oplus \beta \cdot g)(x)=\sup _{y \in \mathbb{R}^{n}} f\left(\frac{x-y}{\alpha}\right)^{\alpha} g\left(\frac{y}{\beta}\right)^{\beta} .
$$

In particularly, when $\alpha=0$ and $\beta>0$, we have $(\alpha \cdot f \oplus \beta \cdot g)(x)=g\left(\frac{x}{\beta}\right)^{\beta}$; when $\alpha>0$ and $\beta=0$, then $(\alpha \cdot f \oplus \beta \cdot g)(x)=f\left(\frac{x}{\alpha}\right)^{\alpha}$; finally, when $\alpha=\beta=0$, we have $(\alpha \cdot f \oplus \beta \cdot g)=I_{\{0\}}$.

Proposition 1.1 grants that $\mathcal{L}$ is closed under the operations of infimal convolution and right scalar multiplication.

Proposition 1.4 ([15]) Let $u$ and $v$ belong both to the same class $\mathcal{L}$, and $\alpha, \beta \geq 0$. Then $u \alpha \square v \beta$ belongs to the same class as $u$ and $v$.

Let $f \in \mathcal{A}$, the support function of $f=e^{-u}$ is defined by

$$
h_{f}(x)=(-\log f(x))^{*}=u^{*}(x),
$$

here the $u^{*}$ is the Legendre transform of $u$. The definition of $h_{f}$ is a proper generalization of the support function $h_{K}$, in fact, one can easily check $h_{\chi_{K}}=h_{K}$ (see $\left.[3,26]\right)$. Specifically, the function $h: \mathcal{A} \rightarrow \mathcal{L}$ has the following properties [27]:

1. $h$ is a bijective map from $\mathcal{A} \rightarrow \mathcal{L}$.

2. $h$ is order preserving: $f \leq g$ if and only if $h_{f} \leq h_{g}$.

3. $h$ is additive: for every $f, g \in \mathcal{A}$ we have $h_{f \oplus g}=h_{f}+h_{g}$.

The following proposition shows that $h_{f}$ is $G L(n)$ covariant.

Proposition 1.5 ([16]) Let $f \in \mathcal{A}$, and $A \in G L(n)$. Then, for $x \in \mathbb{R}^{n}$,

$$
h_{f \circ A}(x)=h_{f}\left(A^{-t} x\right) .
$$

Let $u, v \in \mathcal{L}$, denote $u_{t}=u \square v t(t>0)$, and $f_{t}=e^{-u_{t}}$. The following lemmas describe the monotonicity and convergence of $u_{t}$ and $f_{t}$, respectively. 
Lemma 1.6 ([15]) Let $f, g \in \mathcal{A}$. For $t>0$, set $u_{t}=u \square(v t), f_{t}=e^{-u_{t}}$, and assume that $v(0)=$ 0 . Then, for every fixed $x \in \mathbb{R}^{n}, u_{t}(x)$ and $f_{t}(x)$ are, respectively, pointwise decreasing and increasing with respect to $t$; in particular

$$
u_{1}(x) \leq u_{t}(x) \leq u(x) \quad \text { and } \quad f(x) \leq f_{t}(x) \leq f_{1}(x) \quad \forall x \in \mathbb{R}^{n} \forall t \in[0,1]
$$

Lemma 1.7 ([15]) Let $u$ and $v$ belong both to the same class $\mathcal{L}$ and, for any $t>0$, set $u_{t}:=$ $u \square(v t)$, assume that $v(0)=0$. Then

1. $\forall x \in \Omega, \lim _{t \rightarrow 0^{+}} u_{t}(x)=u(x)$;

2. $\forall E \subset \subset \Omega, \lim _{t \rightarrow 0^{+}} \nabla u_{t}(x)=\nabla u$ uniformly on $E$.

Lemma 1.8 ([15]) Let $u$ and $v$ belong both to the same class $\mathcal{L}$, for any $t>0$, let $u_{t}:=u \square(v t)$. Then $\forall x \in \operatorname{int}\left(\Omega_{t}\right)$, and $\forall t>0$,

$$
\frac{d}{d t}\left(u_{t}(x)\right)=-\psi\left(\nabla u_{t}(x)\right)
$$

where $\psi:=v^{*}$.

\section{Projection of functions onto linear subspace}

Let $\mathcal{G}_{i, n}(0 \leq i \leq n)$ be the Grassmannian manifold of $i$-dimensional linear subspace of $\mathbb{R}^{n}$. The elements of $\mathcal{G}_{i, n}$ will usually be denoted by $\xi_{i}$ and $\xi_{i}^{\perp}$ stands for the orthogonal complement of $\xi_{i}$ which is a $(n-i)$-dimensional subspace of $\mathbb{R}^{n}$. Let $\xi_{i} \in \mathcal{G}_{i, n}$ and $f: \mathbb{R}^{n} \rightarrow$ $\mathbb{R}$. The projection of $f$ onto $\xi_{i}$ is defined by (see $[20,22]$ )

$$
\left.f\right|_{\xi_{i}}(x):=\max \left\{f(y): y \in x+\xi_{i}{ }^{\perp}\right\},\left.\quad \forall x \in \Omega\right|_{\xi_{i}} .
$$

Here $\xi_{i}^{\perp}$ is the orthogonal complement of $\xi_{i}$ in $\mathbb{R}^{n},\left.\Omega\right|_{\xi_{i}}$ is the projection of $\Omega$ onto $\xi_{i}$. By the definition of the log-concave function $f=e^{-u}$, for every $\left.x \in \Omega\right|_{\xi_{i}}$, one can rewrite (2.1) as

$$
\left.f\right|_{\xi_{i}}(x)=\exp \left\{\max \left\{-u(y): y \in x+\xi_{i}^{\perp}\right\}\right\}=e^{-\left.u\right|_{\xi_{i}}}(x)
$$

As regards the "sum" and "multiplication" of $f$, we say that the projection keeps the structure on $\mathbb{R}^{n}$. In other words, we have the following proposition.

Proposition 2.1 Letf, $g \in \mathcal{A}, \xi_{i} \in \mathcal{G}_{i, n}$ and $\alpha, \beta \geq 0$. Then

$$
\left.(\alpha \cdot f \oplus \beta \cdot g)\right|_{\xi_{i}}=\left.\left.\alpha \cdot f\right|_{\xi_{i}} \oplus \beta \cdot g\right|_{\xi_{i}} .
$$

Proof Let $f, g \in \mathcal{A}$, set $x_{1}, x_{2}, x \in \xi_{i}$ such that $x=\alpha x_{1}+\beta x_{2}$. Then, by the definition of the projection, we have

$$
\begin{aligned}
\left.(\alpha \cdot f \oplus \beta \cdot g)\right|_{\xi_{i}}(x) & \geq(\alpha \cdot f \oplus \beta \cdot g)\left(\alpha x_{1}+\beta x_{2}+\xi_{i}^{\perp}\right) \\
& \geq f\left(x_{1}+\xi_{i}^{\perp}\right)^{\alpha} g\left(x_{2}+\xi_{i}^{\perp}\right)^{\beta} .
\end{aligned}
$$


Taking the supremum of the second right hand inequality over all $\xi_{i}^{\perp}$ we obtain $(\alpha \cdot f \oplus \beta$. $g)\left.\right|_{\xi_{i}} \geq\left.\left.\alpha \cdot f\right|_{\xi_{i}} \oplus \beta \cdot g\right|_{\xi_{i}}$. On the other hand, for $x \in \xi_{i}$, any $x_{1}, x_{2} \in \xi_{i}$ such $x_{1}+x_{2}=x$ then

$$
\begin{aligned}
\left(\left.\left.\alpha \cdot f\right|_{\xi_{i}} \oplus \beta \cdot g\right|_{\xi_{i}}\right)(x) & =\sup _{x_{1}+x_{2}=x}\left(\left.f\right|_{\xi_{i}}\right)^{\alpha}\left(\frac{x_{1}}{\alpha}\right)\left(\left.g\right|_{\xi_{i}}\right)^{\beta}\left(\frac{x_{2}}{\beta}\right) \\
& =\sup _{x_{1}+x_{2}=x}\left\{\max \left\{f^{\alpha}\left(\frac{x_{1}}{\alpha}+\xi_{i}^{\perp}\right)\right\} \max \left\{g^{\beta}\left(\frac{x_{2}}{\beta}+\xi_{i}^{\perp}\right)\right\}\right\} \\
& \geq \sup _{x_{1}+x_{2}=x}\left\{\max \left(f^{\alpha}\left(\frac{x_{1}}{\alpha}+\xi_{i}^{\perp}\right) g^{\beta}\left(\frac{x_{2}}{\beta}+\xi_{i}^{\perp}\right)\right)\right\} \\
& =\max \left\{(\alpha \cdot f \oplus \beta \cdot g)\left(x_{1}+x_{2}+\xi_{i}^{\perp}\right)\right\} \\
& =\left.(\alpha \cdot f \oplus \beta \cdot g)\right|_{\xi_{i}}(x) .
\end{aligned}
$$

Since $f, g \geq 0$, the inequality $\max \{f \cdot g\} \leq \max \{f\} \cdot \max \{g\}$ holds. So we complete the proof.

Proposition 2.2 Let $\xi_{i} \in \mathcal{G}_{i, n}, f$ and $g$ are functions on $\mathbb{R}^{n}$, such that $f(x) \leq g(x)$ holds. Then

$$
\left.f\right|_{\xi_{i}} \leq\left. g\right|_{\xi_{i}}
$$

holds for any $x \in \xi_{i}$.

Proof For $y \in x+\xi_{i}^{\perp}$, since $f(y) \leq g(y)$, then $f(y) \leq \max \left\{g(y): y \in x+\xi_{i}^{\perp}\right\}$. So $\max \{f(y)$ : $\left.y \in x+L_{i}^{\perp}\right\} \leq \max \left\{g(y): y \in x+\xi_{i}^{\perp}\right\}$, by the definition of the projection, we complete the proof.

For the convergence of $f$ we have the following.

Proposition 2.3 Let $\left\{f_{i}\right\}$ be functions such that $\lim _{n \rightarrow \infty} f_{n}=f_{0}$. Let $\xi_{i} \in \mathcal{G}_{i, n}$, then $\lim _{n \rightarrow \infty}\left(\left.f_{n}\right|_{\xi_{i}}\right)=\left.f_{0}\right|_{\xi_{i}}$.

Proof Since $\lim _{n \rightarrow \infty} f_{n}=f_{0}$, it means that, for $\forall \epsilon>0$, there exists $N_{0}, \forall n>N_{0}$, such that $f_{0}-\epsilon \leq f_{n} \leq f_{0}+\epsilon$. By the monotonicity of the projection, we have $\left.f_{0}\right|_{\xi_{i}}-\epsilon \leq\left. f_{n}\right|_{\xi_{i}} \leq\left. f_{0}\right|_{\xi_{i}}+\epsilon$. Hence each $\left\{\left.f_{n}\right|_{\xi_{i}}\right\}$ has a convergent subsequence, we denote it also by $\left\{\left.f_{n}\right|_{\xi_{i}}\right\}$, converging to some $\left.f_{0}^{\prime}\right|_{\xi_{i}}$. Then, for $x \in \xi_{i}$, we have

$$
\left.f_{0}\right|_{\xi_{i}}(x)-\epsilon \leq\left. f_{0}^{\prime}\right|_{\xi_{i}}(x)=\lim _{n \rightarrow \infty}\left(\left.f_{n}\right|_{\xi_{i}}\right)(x) \leq\left. f_{0}\right|_{\xi_{i}}(x)+\epsilon .
$$

By the arbitrariness of $\epsilon$ we have $\left.f_{0}^{\prime}\right|_{\xi_{i}}=\left.f_{0}\right|_{\xi_{i}}$, so we complete the proof.

Combining with Proposition 2.3 and Proposition 1.7, it is easy to obtain the following proposition.

Proposition 2.4 Let $u$ and $v$ belong both to the same class $\mathcal{L}, \Omega \in \mathbb{R}^{n}$ be the domain of $u$, for any $t>0$, set $u_{t}=u \square(v t)$. Assume that $v(0)=0$ and $\xi_{i} \in \mathcal{G}_{i, n}$, then

1. $\left.\forall x \in \Omega\right|_{\xi_{i}},\left.\lim _{t \rightarrow 0^{+}} u_{t}\right|_{\xi_{i}}(x)=\left.u\right|_{\xi_{i}}(x)$, 
2. $\forall x \in \operatorname{int}\left(\left.\Omega\right|_{\xi_{i}}\right),\left.\lim _{t \rightarrow 0^{+}} \nabla u_{t}\right|_{\xi_{i}}=\left.\nabla u\right|_{\xi_{i}}$.

Now let us introduce some facts about the functions $u_{t}=u \square(v t)$ with respect to the parameter $t$.

Lemma 2.5 Let $\xi_{i} \in \mathcal{G}_{i, n}$, $u$ and $v$ belong both to the same class $\mathcal{L}, u_{t}:=u \square(v t)(t>0)$ and $\Omega_{t}$ be the domain of $u_{t}$. Then, for $\left.x \in \Omega_{t}\right|_{\xi_{i}}$,

$$
\frac{d}{d t}\left(\left.u_{t}\right|_{\xi_{i}}\right)(x)=-\psi\left(\nabla\left(\left.u_{t}\right|_{\xi_{i}}\right)(x)\right)
$$

where $\psi:=\left.v^{*}\right|_{\xi_{i}}$.

Proof Set $D_{t}:=\left.\Omega_{t}\right|_{\xi_{i}} \subset \xi_{i}$, for fixed $x \in \operatorname{int}\left(D_{t}\right)$, the map $t \rightarrow \nabla\left(\left.u_{t}\right|_{\xi_{i}}\right)(x)$ is differentiable on $(0,+\infty)$. Indeed, by the definition of Fenchel conjugate and the definition of projection $u$, it is easy to see that $\left(\left.u\right|_{\xi_{i}}\right)^{*}=\left.u^{*}\right|_{\xi_{i}}$ and $\left.(u \square u t)\right|_{\xi_{i}}=\left.\left.u\right|_{\xi_{i}} \square u t\right|_{\xi_{i}}$ hold. Lemma 1.4 and the property of the projection grant the differentiability. Set $\varphi:=\left.u^{*}\right|_{\xi_{i}}$ and $\psi:=\left.v^{*}\right|_{\xi_{i}}$, and $\varphi_{t}=$ $\varphi+t \psi$, then $\varphi_{t}$ belongs to the class $\mathcal{C}_{+}^{2}$ on $\xi_{i}$. Then $\nabla^{2} \varphi_{t}=\nabla^{2} \varphi+t \nabla^{2} \psi$ is nonsingular on $\xi_{i}$. So the equation

$$
\nabla \varphi(y)+t \nabla \psi(y)-x=0
$$

locally defines a map $y=y(x, t)$ which is of class $\mathcal{C}^{1}$. By Proposition 1.3, $\nabla\left(\left.u_{t}\right|_{\xi_{i}}\right)$ is the inverse map of $\nabla \varphi_{t}$, that is, $\nabla \varphi_{t}\left(\nabla\left(\left.u_{t}\right|_{\xi_{i}}(x)\right)=x\right.$, which means that, for every $x \in \operatorname{int}\left(D_{t}\right)$ and every $t>0, t \rightarrow \nabla\left(\left.u_{t}\right|_{\xi_{i}}\right)$ is differentiable. Using Eq. (1.2) again, we have

$$
\left.u_{t}\right|_{\xi_{i}}(x)=\left\langle x, \nabla\left(\left.u_{t}\right|_{\xi_{i}}\right)(x)\right\rangle-\varphi_{t}\left(\nabla\left(\left.u_{t}\right|_{\xi_{i}}\right)(x)\right), \quad \forall x \in \operatorname{int}\left(D_{t}\right) .
$$

Moreover, note that $\varphi_{t}=\varphi+t \psi$ and we have

$$
\begin{aligned}
\left.u_{t}\right|_{\xi_{i}}(x) & =\left\langle x, \nabla\left(\left.u_{t}\right|_{\xi_{i}}\right)(x)\right\rangle-\varphi\left(\nabla\left(u_{t} \mid \xi_{i}\right)(x)\right)-t \psi\left(\nabla\left(\left.u_{t}\right|_{\xi_{i}}\right)(x)\right) \\
& =\left.u_{t}\right|_{\xi_{i}}\left(\nabla\left(\left.u_{t}\right|_{\xi_{i}}\right)(x)\right)-t \psi\left(\nabla\left(\left.u_{t}\right|_{\xi_{i}}\right)(x)\right) .
\end{aligned}
$$

Taking the differential of the above formally we obtain

$$
\frac{d}{d t}\left(\left.u_{t}\right|_{\xi_{i}}\right)(x)=-\psi\left(\nabla\left(\left.u_{t}\right|_{\xi_{i}}\right)(x)\right) .
$$

Then we complete the proof.

\section{Inequality for functional mixed quermassintegral}

A function $f \in \mathcal{A}$ is non-degenerate and integrable if and only if $\lim _{\|x\| \rightarrow+\infty} \frac{u(x)}{\|x\|}=+\infty$. Then, let $\mathcal{L}^{\prime}=\left\{u \in \mathcal{L}: u \in \mathcal{C}_{+}^{2}\left(\mathbb{R}^{n}\right), \lim _{\|x\| \rightarrow+\infty} \frac{u(x)}{\|x\|}=+\infty\right\}$, and $\mathcal{A}^{\prime}=\left\{f: \mathbb{R}^{n} \rightarrow(0,+\infty]: f=\right.$ $\left.e^{-u}, u \in \mathcal{L}^{\prime}\right\}$.

Definition 3.1 Let $f \in \mathcal{A}^{\prime}, \xi_{i} \in \mathcal{G}_{i, n}(i=1,2, \ldots, n)$, and $\left.x \in \Omega\right|_{\xi_{i}}$. The $i$ th total mass of $f$ is defined as

$$
J_{i}(f):=\left.\int_{\xi_{i}} f\right|_{\xi_{i}}(x) d x
$$


where $\left.f\right|_{\xi_{i}}$ is the projection of $f$ onto $\xi_{i}$ defined by (2.1), $d x$ is the $i$-dimensional volume element in $\xi_{i}$.

Remark 3.1 (1) The definition of the $J_{i}(f)$ follows the $i$-dimensional volume of the projection of a convex body. If $i=0$, we define $J_{0}(f):=\omega_{n}$, the volume of the unit ball in $\mathbb{R}^{n}$, for completeness.

(2) When one takes $f=\chi_{K}$, the characteristic function of a convex body $K$, one has $J_{i}(f)=V_{i}(K)$, the $i$-dimensional volume in $\xi_{i}$.

Definition 3.2 Let $f \in \mathcal{A}^{\prime}$. Set $\xi_{i} \in \mathcal{G}_{i, n}$ be a linear subspace and, for any $\left.x \in \Omega\right|_{\xi_{i}}$, the $i$ th functional Quermassintegrals of $f$ (or the $i$-dimensional mean projection mass of $f$ ) is defined as

$$
W_{n-i}(f):=\frac{\omega_{n}}{\omega_{i}} \int_{\mathcal{G}_{i, n}} J_{i}(f) d \mu\left(\xi_{i}\right), \quad i=1,2, \ldots, n,
$$

where $J_{i}(f)$ is the $i$ th total mass of $f$ defined by $(3.1), d \mu\left(\xi_{i}\right)$ is the normalized Haar measure on $\mathcal{G}_{i, n}$.

Remark 3.2 (1) The definition of the $W_{i}(f)$ follows the definition of the $i$ th Quermassintegral $W_{i}(K)$, that is, the $i$ th mean total mass of $f$ on $\mathcal{G}_{i, n}$. Also in the recent paper of Bobkov, Colesanti and Fragala [10], the authors give the same definition by defining the Quermassintegral of the support set for the quasi-concave functions.

(2) When $i$ equals $n$ in (3.2), we have $W_{0}(f)=\int_{\mathbb{R}^{n}} f(x) d x=J(f)$.

(3) From the definition of the Quermassintegrals $W_{i}(f)$, the following properties are obtained (see also [10]):

- Positivity. $0 \leq W_{i}(f) \leq+\infty$.

- Monotonicity. $W_{i}(f) \leq W_{i}(g)$, if $f \leq g$.

- Generally speaking, the $W_{i}(f)$ has no homogeneity under dilations. That is, $W_{i}(\lambda \cdot f)=\lambda^{n-i} W_{i}\left(f^{\lambda}\right)$, where $\lambda \cdot f(x)=\lambda f(x / \lambda), \lambda>0$.

Definition 3.3 Let $f, g \in \mathcal{A}^{\prime}, \oplus$ and · denote the operations of "sum" and "multiplication" in $\mathcal{A}^{\prime}, W_{i}(f)$ be the $i$ th Quermassintegrals of $f$. Whenever the following limit exists:

$$
W_{i}(f, g)=\frac{1}{(n-i)} \lim _{t \rightarrow 0^{+}} \frac{W_{i}(f \oplus t \cdot g)-W_{i}(f)}{t}, \quad i=0,1, \ldots, n-1,
$$

we denote it by $W_{i}(f, g)$, and call it the first variation of $W_{i}$ at $f$ along $g$, or the $i$ th functional mixed Quermassintegrals of $f$ and $g$.

Remark 3.3 Let $f=\chi_{K}$ and $g=\chi_{L}$, with $K, L \in \mathcal{K}^{n}$. In this case $W_{i}(f \oplus t \cdot g)=W_{i}(K+t L)$, then $W_{i}(f, g)=W_{i}(K, L)$.

The following is devoted to proving that $W_{i}(f, g)$ exists under the fairly weak hypothesis. First, we prove that the first variation of $i$-dimensional total mass of $f$ is translation invariant.

Lemma 3.4 Let $\xi_{i} \in \mathcal{G}_{i, n}, f=e^{-u}$ and $g=e^{-v}$ are integrable log-concave functions in $\mathcal{A}^{\prime}$. Let $c=\left.\inf u\right|_{\xi_{i}}=: u(0), d=\left.\inf v\right|_{\xi_{i}}:=v(0)$, and set $\widetilde{u}_{i}(x)=\left.u\right|_{\xi_{i}}(x)-c, \widetilde{v}_{i}(x)=\left.v\right|_{\xi_{i}}(x)-d, \widetilde{\varphi}_{i}(y)=$ 
$\left(\widetilde{u}_{i}\right)^{*}(y), \widetilde{\psi}_{i}(y)=\left(\widetilde{v}_{i}\right)^{*}(y)$, and $\widetilde{f}_{i}=e^{-\widetilde{u}_{i}}, \widetilde{g}_{i}=e^{-\widetilde{v}_{i}},\left.\widetilde{f}_{t}\right|_{i}=\tilde{f} \oplus t \cdot \widetilde{g}$. Then, if

$$
\lim _{t \rightarrow 0^{+}} \frac{J_{i}\left(\widetilde{f}_{t}\right)-J_{i}(\widetilde{f})}{t}=\int_{\xi_{i}} \widetilde{\psi}_{i} d \mu_{i}(\widetilde{f})
$$

then we have

$$
\lim _{t \rightarrow 0^{+}} \frac{J_{i}\left(f_{t}\right)-J_{i}(f)}{t}=\int_{\xi_{i}} \psi_{i} d \mu_{i}(f) .
$$

Proof By the construction, we have $\widetilde{u}_{i}(0)=0, \widetilde{v}_{i}(0)=0$, and $\widetilde{v}_{i} \geq 0, \widetilde{\varphi}_{i} \geq 0, \widetilde{\psi}_{i} \geq 0$. Further, we have $\widetilde{\psi}_{i}(y)=\psi_{i}(y)+d$, and $\widetilde{f}_{i}=e^{c} f_{i}$. Then we have

$$
\lim _{t \rightarrow 0^{+}} \frac{J_{i}\left(\widetilde{f}_{t}\right)-J_{i}(\widetilde{f})}{t}=\int_{\xi_{i}} \widetilde{\psi}_{i} d \mu_{i}(\widetilde{f})=e^{c} \int_{\xi_{i}} \psi_{i} d \mu_{i}(f)+d e^{c} \int_{\xi_{i}} d \mu_{i}(f) .
$$

On the other hand, since $f_{i} \oplus t \cdot g_{i}=e^{-(c+d t)}\left(\widetilde{f}_{i} \oplus t \cdot \widetilde{g}_{i}\right)$, we have $J_{i}(f \oplus t \cdot g)=e^{-(c+d t)} J_{i}\left(\widetilde{f}_{i} \oplus\right.$ $\left.t \cdot \widetilde{g}_{i}\right)$. By derivation of both sides of the above formula, we obtain

$$
\begin{aligned}
\lim _{t \rightarrow 0^{+}} & \frac{J_{i}(f \oplus t \cdot g)-J_{i}(f)}{t} \\
= & -d e^{-c} \lim _{t \rightarrow 0^{+}} J_{i}\left(\widetilde{f}_{i} \oplus t \widetilde{g}_{i}\right) d x+e^{-c} \lim _{t \rightarrow 0^{+}}\left[\frac{J_{i}\left(\widetilde{f}_{t}\right)-J_{i}(\widetilde{f})}{t}\right] \\
= & -d e^{-c} J_{i}\left(\widetilde{f}_{i}\right)+\int_{\xi_{i}} \psi_{i} d \mu_{i}(f)+d \int_{\xi_{i}} d \mu_{i}(f) \\
= & \int_{\xi_{i}} \psi_{i} d \mu_{i}(f) .
\end{aligned}
$$

So we complete the proof.

Theorem 3.5 Let $f, g \in \mathcal{A}^{\prime}$, with $-\infty \leq \inf (\log g) \leq+\infty$, and $W_{i}(f)>0$. Then $W_{j}(f, g)$ is differentiable at $f$ along $g$, and

$$
W_{j}(f, g) \in[-k,+\infty],
$$

where $k=\max \{d, 0\} W_{i}(f)$.

Proof Let $\xi_{i} \in \mathcal{G}_{i, n}$, since $\left.u\right|_{\xi_{i}}:=-\log \left(\left.f\right|_{\xi_{i}}\right)=-\left.(\log f)\right|_{\xi_{i}}$ and $\left.v\right|_{\xi_{i}}:=-\log \left(\left.g\right|_{\xi_{i}}\right)=-\left.(\log f)\right|_{\xi_{i}}$. By the definition of $f_{t}$ and the Proposition 2.1 we obtain

$$
\left.f_{t}\right|_{\xi_{i}}=\left.(f \oplus t \cdot g)\right|_{\xi_{i}}=\left.\left.f\right|_{\xi_{i}} \oplus t \cdot g\right|_{\xi_{i}} .
$$

Notice that $\left.v\right|_{\xi_{i}}(0)=v(0)$, set $d:=v(0),\left.\widetilde{v}\right|_{\xi_{i}}(x):=\left.v\right|_{\xi_{i}}(x)-d,\left.\widetilde{g}\right|_{\xi_{i}}(x):=e^{-\left.\widetilde{v}\right|_{i}(x)},\left.\widetilde{f}_{t}\right|_{\xi_{i}}:=\left.f\right|_{\xi_{i}} \oplus$ $\left.t \cdot \widetilde{g}\right|_{\xi_{i}}$. Without loss of generality, we may assume $\inf (v)=v(0)$. Lemma 1.6 says that, for every $x \in \xi_{i}$,

$$
\left.f\right|_{\xi_{i}} \leq\left.\tilde{f}_{t}\right|_{\xi_{i}} \leq\left.\tilde{f}_{1}\right|_{\xi_{i}}, \quad \forall x \in \mathbb{R}^{n}, \forall t \in[0,1] .
$$


Then there exists $\left.\widetilde{f}\right|_{\xi_{i}}(x):=\left.\lim _{t \rightarrow 0^{+}} \widetilde{f}_{t}\right|_{\xi_{i}}(x)$, moreover, $\left.\widetilde{f}\right|_{\xi_{i}}(x) \geq\left. f\right|_{\xi_{i}}(x)$ and $\left.\widetilde{f}_{t}\right|_{\xi_{i}}$ is pointwise decreasing as $t \rightarrow 0^{+}$. By Lemma 1.1 and Proposition 1.4, one shows that

$$
\left.\left.f\right|_{\xi_{i}} \oplus t \cdot \widetilde{g}\right|_{\xi_{i}} \in A^{\prime}, \quad \forall t \in[0,1]
$$

Then $J_{i}(f) \leq J_{i}\left(\widetilde{f}_{t}\right) \leq J_{i}\left(\widetilde{f}_{1}\right)$, and, $-\infty \leq J_{i}(f), J_{i}\left(\widetilde{f}_{1}\right)<\infty$. Hence, by the monotonicity and convergence, we have $\lim _{t \rightarrow 0^{+}} W_{i}\left(\widetilde{f}_{t}\right)=W_{i}(\widetilde{f})$. In fact, by definition we have $\left.\widetilde{f}_{t}\right|_{\xi_{i}}(x)=$ $e^{-\inf \left\{u\left|\xi_{i}(x-y)+t v\right| \xi_{i}\left(\frac{y}{t}\right)\right\}}$, and

$$
-\inf \left\{\left.u\right|_{\xi_{i}}(x-y)+\left.t v\right|_{\xi_{i}}\left(\frac{y}{t}\right)\right\} \leq-\left.\inf u\right|_{\xi_{i}}(x-y)-\left.t \inf v\right|_{\xi_{i}}\left(\frac{y}{t}\right) .
$$

Note that $-\infty \leq \inf \left(\left.v\right|_{\xi_{i}}\right) \leq+\infty$, then $-\left.\inf u\right|_{\xi_{i}}(x-y)-\left.t \inf v\right|_{\xi_{i}}\left(\frac{y}{t}\right)$ is a continuous function of variable $t$, then

$$
\left.\widetilde{f}\right|_{\xi_{i}}(x):=\left.\lim _{t \rightarrow 0^{+}} \widetilde{f}_{t}\right|_{\xi_{i}}(x)=\left.f\right|_{\xi_{i}}(x)
$$

Moreover, $W_{i}\left(\widetilde{f}_{t}\right)$ is a continuous function of $t(t \in[0,1])$, then $\lim _{t \rightarrow 0^{+}} W_{i}\left(\widetilde{f}_{t}\right)=W_{i}(f)$. Since $\left.f_{t}\right|_{\xi_{i}}=\left.e^{-d t} \widetilde{f}\right|_{\xi_{i}}(x)$, we have

$$
\frac{W_{i}\left(f_{t}\right)-W_{i}(f)}{t}=W_{i}(f) \frac{e^{-d t}-1}{t}+e^{-d t} \frac{W_{i}\left(\widetilde{f}_{t}\right)-W_{i}(f)}{t} .
$$

Since $\left.\widetilde{f}_{t}\right|_{\xi_{i}} \geq\left. f\right|_{\xi_{i}}$, we have the following two cases:

$$
\exists t_{0}>0: W_{i}\left(\widetilde{f}_{t_{0}}\right)=W_{i}(f) \quad \text { or } \quad W_{i}\left(\widetilde{f}_{t}\right)=W_{i}(f) \quad \forall t>0 .
$$

For the first case, since $W_{i}\left(\widetilde{f}_{t}\right)$ is a monotone increasing function of $t, W_{i}\left(\widetilde{f}_{t}\right)=W_{i}(f)$ for every $t \in\left[0, t_{0}\right]$. Hence we have

$$
\lim _{t \rightarrow 0^{+}} \frac{W_{i}\left(f_{t}\right)-W_{i}(f)}{t}=-d W_{i}(f)
$$

the statement of the theorem holds true.

In the latter case, since $\left.\widetilde{f}_{t}\right|_{\xi_{i}}$ is an increasing nonnegative function, $\log \left(W_{i}\left(\widetilde{f}_{t}\right)\right)$ is an increasing concave function of $t$. Then

$$
\exists \frac{\log \left(W_{i}\left(\widetilde{f}_{t}\right)\right)-\log \left(W_{i}(f)\right)}{t} \in[0,+\infty]
$$

On the other hand,

$$
\left.\log ^{\prime}\left(W_{i}\left(\widetilde{f}_{t}\right)\right)\right|_{t=0}=\lim _{t \rightarrow 0^{+}} \frac{\log \left(W_{i}\left(\widetilde{f}_{t}\right)\right)-\log \left(W_{i}(f)\right)}{W_{i}\left(\widetilde{f}_{t}\right)-W_{i}(f)}=\frac{1}{W_{i}(f)} .
$$

Then

$$
\lim _{t \rightarrow 0^{+}} \frac{W_{i}\left(\widetilde{f}_{t}\right)-W_{i}(f)}{\log \left(W_{i}\left(\widetilde{f}_{t}\right)\right)-\log \left(W_{i}(f)\right)}=W_{i}(f)>0 .
$$


From the above we infer that

$$
\exists \lim _{t \rightarrow 0^{+}} \frac{W_{i}\left(\widetilde{f}_{t}\right)-W_{i}(f)}{t} \in[0,+\infty] .
$$

Combining the above formulas we obtain

$$
\lim _{t \rightarrow 0^{+}} \frac{W_{i}\left(f_{t}\right)-W_{i}(f)}{t} \in\left[-\max \{d, 0\} W_{i}(f),+\infty\right]
$$

So we complete the proof.

Corollary 3.6 Let $f \in \mathcal{A}^{\prime}$, then

$$
W_{i}(f, f)=W_{i}(f)+\frac{1}{(n-i)} W_{i}(f \log f)
$$

Proof Since $f \in \mathcal{A}^{\prime}$, we have $\left.f\right|_{\xi_{i}} \in \mathcal{A}^{\prime} . u \square u t=u(1+t)$, then $\left.u \square u t\right|_{\xi_{i}}=\left.u(1+t)\right|_{\xi_{i}}$. So

$$
\begin{aligned}
\frac{J_{i}(f \oplus t \cdot f)-J_{i}(f)}{t} & =\frac{1}{t}\left[(1+t)^{i} \int_{\xi_{i}} e^{-\left.(1+t) u\right|_{\xi_{i}}} d x-\int_{\xi_{i}} e^{-\left.u\right|_{\xi_{i}}} d x\right] \\
& =\left[\frac{(1+t)^{i}-1}{t}\right] \int_{\xi_{i}} e^{-\left.(1+t) u\right|_{\xi_{i}}} d x+\int_{\xi_{i}} e^{-\left.u\right|_{\xi_{i}}}\left(\frac{e^{-t u \mid \xi_{i}}-1}{t}\right) d x .
\end{aligned}
$$

Now taking the limit when $t \rightarrow 0^{+}$, we obtain

$$
\lim _{t \rightarrow 0^{+}} \frac{J_{i}(f \oplus t \cdot f)-J_{i}(f)}{t}=i J_{i}(f)+\left.\left.\int_{\xi_{i}} f\right|_{\xi_{i}} \log f\right|_{\xi_{i}} d x .
$$

Then we have

$$
\begin{aligned}
\lim _{t \rightarrow 0^{+}} & \frac{W_{i}(f \oplus t \cdot f)-W_{i}(f)}{t} \\
= & \frac{\omega_{n}}{\omega_{n-i}} \int_{\mathcal{G}_{n-i, n}} \lim _{t \rightarrow 0^{+}} \frac{J_{n-i}(f \oplus t \cdot f)-J_{n-i}(f)}{t} d \mu\left(\xi_{n-i}\right) \\
= & \frac{\omega_{n}}{\omega_{n-i}} \int_{\mathcal{G}_{n-i, n}}\left[(n-i) J_{n-i}(f)+\left.\left.\int_{\xi_{n-i}} f\right|_{\xi_{n-i}} \log f\right|_{\xi_{n-i}} d x\right] d \mu\left(\xi_{n-i}\right) \\
= & (n-i) W_{i}(f)+\left.\left.\frac{\omega_{n}}{\omega_{n-i}} \int_{\mathcal{G}_{n-i, n}} \int_{\xi_{n-i}} f\right|_{\xi_{n-i}} \log f\right|_{\xi_{n-i}} d x d \mu\left(\xi_{n-i}\right) \\
= & (n-i) W_{i}(f)+\left.\frac{\omega_{n}}{\omega_{n-i}} \int_{\mathcal{G}_{n-i, n}} \int_{\xi_{n-i}}(f \log f)\right|_{\xi_{n-i}} d x d \mu\left(\xi_{n-i}\right) \\
= & (n-i) W_{i}(f)+W_{i}(f \log f) .
\end{aligned}
$$

Here we use the $\left.(f \log f)\right|_{\xi_{i}}=\left.\left.f\right|_{\xi_{i}} \log f\right|_{\xi_{i}}$, due to the $f$ and $\log f$ being increasing nonnegative functions. Then by the definition we obtain $W_{i}(f, f)=W_{i}(f)+\frac{1}{(n-i)} W_{i}(f \log f)$. Then we complete the proof.

The following lemma is useful in proving Minkowski's first inequality for Quermassintegrals. 
Lemma 3.7 Let $f, g \in \mathcal{A}^{\prime}$, and $0<t<1$. Then

$$
\lim _{t \rightarrow 0^{+}} \frac{W_{i}((1-t) \cdot f \oplus t \cdot g)-W_{i}(f)}{t}=(n-i)\left[W_{i}(f, g)-W_{i}(f, f)\right] .
$$

Proof First by Lemma 3.4, without loss of generality, we may assume that the function $v=-\log g$ satisfies the condition $v(0)=0$. For $t \in(0,1)$, letting $s(t)=\frac{t}{1-t}$, by $(1.9)$ we obtain $(1-t) \cdot f \oplus t \cdot g=(1-t) \cdot(f \oplus s(t) \cdot g)$. Let $f_{s(t)}=f \oplus s(t) \cdot g$, then we have

$$
\begin{aligned}
\frac{W_{i}((1-t) \cdot f \oplus t \cdot g)-W_{i}(f)}{t}= & \frac{W_{i}\left((1-t) \cdot f_{s(t)}\right)-W_{i}\left(f_{s(t)}\right)}{t} \\
& +\frac{W_{i}\left(f_{s(t)}\right)-W_{i}(f)}{t} .
\end{aligned}
$$

Concerning the first term of the right hand side (3.13), by Lemma 1.6 we know that the function $f_{s(t)}(x)$ converges decreasingly to some pointwise limit $f(x)$ as $t \rightarrow 0^{+}$, since $s(t) \rightarrow$ $0^{+}$as $t \rightarrow 0^{+}$. In fact, we have $\lim _{t \rightarrow 0^{+}} f_{s(t)}(x)=\lim _{t^{\prime} \rightarrow 0} f_{t^{\prime}}(x)=f(x)$. Then we obtain

$$
\begin{aligned}
\lim _{t \rightarrow 0^{+}} \frac{W_{i}\left((1-t) \cdot f_{s(t)}\right)-W_{i}\left(f_{s(t)}\right)}{t} & =\lim _{t \rightarrow 0^{+}} \frac{W_{i}((1-t) \cdot f)-W_{i}(f)}{t} \\
& =-(n-i) W_{i}(f, f) .
\end{aligned}
$$

Concerning the second term, we have

$$
\begin{aligned}
\lim _{t \rightarrow 0^{+}} \frac{W_{i}\left(f_{s(t)}\right)-W_{i}(f)}{t} & =\lim _{t \rightarrow 0^{+}} \frac{W_{i}(f \oplus s(t) \cdot g)-W_{i}(f)}{t} \\
& =\lim _{t \rightarrow 0^{+}} \frac{W_{i}(f \oplus s(t) \cdot g)-W_{i}(f)}{s(t)} \cdot \frac{s(t)}{t} \\
& =(n-i) W_{i}(f, g) .
\end{aligned}
$$

Then one can show the conclusion by combining with (3.14) and (3.15).

Now we give the proof of Theorem 0.1 .

Proof of Theorem 0.1 Let $0 \leq t \leq 1$, we construct a function

$$
\Psi(t)=\log \left(W_{i}((1-t) \cdot f \oplus t \cdot g)\right)
$$

In fact, for $f, g, h \in \mathcal{A}^{\prime}$ and $0 \leq t \leq 1$,

$$
\begin{aligned}
\left.h\right|_{\xi_{i}}(z) & =\left(\left.\left.(1-t) \cdot f\right|_{\xi_{i}} \oplus t \cdot g\right|_{\xi_{i}}\right)(z) \\
& =\sup \left\{\left.\left.f\right|_{\xi_{i}}(x)^{1-t} g\right|_{\xi_{i}}(y)^{t}:(1-t) x+t y=z\right\} \\
& \geq\left\{\left.\left.f\right|_{\xi_{i}}(x)^{1-t} g\right|_{\xi_{i}}(y)^{t}:(1-t) x+t y=z\right\} .
\end{aligned}
$$

By the Prékopa-Leindler inequality, for $0 \leq t \leq 1$, we have

$$
\left.\int_{\xi_{i}} h\right|_{\xi_{i}} d z \geq\left(\left.\int_{\xi_{i}} f\right|_{\xi_{i}}(x) d x\right)^{1-t}\left(\left.\int_{\xi_{i}} g\right|_{\xi_{i}}(y) d y\right)^{t}
$$


That means that

$$
J_{i}(h) \geq J_{i}(f)^{1-t} J_{i}(g)^{t}
$$

Taking the integral of both sides of (3.16) on $\mathcal{G}_{i, n}$ with measure $\mu\left(\xi_{i}\right)$, by the PrékopaLeindler inequality once again, we obtain

$$
W_{i}((1-t) \cdot f \oplus t \cdot g) \geq W_{i}(f)^{1-t} W_{i}(g)^{t} .
$$

Since $\Psi(t):=\log \left(W_{i}((1-t) \cdot f \oplus t \cdot g)\right)$, we conclude that $\Psi(t)$ is concave on $[0,1]$. Then

$$
\frac{\Psi(t)-\Psi(0)}{t} \geq \Psi(1)-\Psi(0), \quad \forall t \in[0,1]
$$

It means that $\left.\Psi(t)^{\prime}\right|_{t=0} \geq \Psi(1)-\Psi(0)$.

By Lemma 3.7, we have

$$
\left.\Psi(t)^{\prime}\right|_{t=0}=\left.\frac{W_{i}((1-t) \cdot f \oplus t \cdot g)^{\prime}}{W_{i}((1-t) \cdot f \oplus t \cdot g)}\right|_{t=0}=\frac{(n-i)\left[W_{i}(f, g)-W_{i}(f, f)\right]}{W_{i}(f)} .
$$

On the other hand, note that $\Psi(1)-\Psi(0)=\log \left(W_{i}(g)\right)-\log \left(W_{i}(f)\right)$. Therefore, we obtain

$$
\frac{(n-i)\left[W_{i}(f, g)-W_{i}(f, f)\right]}{W_{i}(f)} \geq \log \left(W_{i}(g)\right)-\log \left(W_{i}(f)\right) .
$$

Then, combining with formula (3.10), we obtain

$$
\begin{aligned}
W_{i}(f, g) & \geq \frac{1}{n-i} W_{i}(f)\left[\log \left(W_{i}(g)-\log W_{i}(f)\right]+W_{i}(f, f)\right. \\
& =W_{i}(f)\left[1+\frac{1}{n-i} \log \frac{W_{i}(g)}{W_{i}(f)}\right]+\frac{1}{n-i} W_{i}(f \log f) .
\end{aligned}
$$

Concerning the equality case, first, assume that $g(x)=f\left(x-x_{0}\right)$, by (3.10) and the invariance of the integral by translation of coordinates, we know that (0.6) holds with equality. On the other hand, if (0.6) holds with equality, by inspection of the above proof, one may see that the inequalities (3.16), (3.17) and (3.18) must hold as equalities. Moreover, whenever inequalities (3.16) and (3.17) hold with equality sign, then (3.18) automatic holds with equality. This entails that the Prékopa-Leindler inequality holds as an equality, therefore $f$ and $g$ must agree up to a translation.

The inequality (0.6) is called the functional Brunn-Minkwoski first inequality for $i$ th mixed Quremassintegrals or functional mixed Quermassintegral inequality. In the following we will give some special case of (0.6).

In fact, we take $f=\chi_{K}$ and $g=\chi_{L}$, with $K, L \in \mathcal{K}^{n}$. In this case $\chi_{K} \oplus t \cdot \chi_{L}=\chi_{K+t L}, J_{i}\left(\chi_{K}\right)=$ $V_{i}(K)$, here $V_{i}$ denotes the $i$-dimensional volume in $\xi_{i}, W_{i}\left(\chi_{K}\right)=W_{i}(K)$, and $W_{i}\left(\chi_{K}, \chi_{L}\right)=$ $W_{i}(K, L)$. Moreover, by (1.6) and (1.7) we have, for any $x$,

$$
f(x) \log f(x)=-e^{-I_{K}(x)} I_{K}(x) \equiv 0 .
$$


Then (0.6) turns out to be

$$
\begin{aligned}
W_{i}(K, L) & \geq W_{i}(K)\left[1+\frac{1}{n-i} \log \frac{W_{i}(L)}{W_{i}(K)}\right] \\
& =W_{i}(K)+\frac{1}{n-i} W_{i}(K) \log \frac{W_{i}(L)}{W_{i}(K)} .
\end{aligned}
$$

We can rewrite the above formula (3.19) equivalently as the following:

$$
\frac{W_{i}(K, L)-W_{i}(K)}{W_{i}(K)} \geq \frac{1}{n-i} \log \frac{W_{i}(L)}{W_{i}(K)} .
$$

By defining the $i$-cone volume probability measure $\bar{V}_{i K}$ similar to the $\bar{V}_{K}$ defined in [11] by Böröczky,

$$
d V_{i K}=\frac{1}{n} h_{K} d S_{i K}
$$

where $d S_{i K}$ is the $i$ th Borel measue on $S^{n-1}$. The normalized $i$-cone volume probability measure $\bar{V}_{i K}$ is defined as

$$
d \bar{V}_{i K}=\frac{1}{W_{i}(K)} d V_{i K}
$$

Then the normalized $i$-mixed Quermassintegrals $\bar{W}_{i}(K, L)$ can be expressed as

$$
\bar{W}_{i}(K, L)=\frac{W_{i}(K, L)}{W_{i}(K)}=\int_{S^{n-1}} \frac{h_{L}}{h_{K}} d \bar{V}_{i K}
$$

Moreover, by the integral representation of $W_{i}(K)$, we have

$$
W_{i}(K)=\frac{1}{n} \int_{S^{n-1}} h_{K} d S_{i K}=\int_{S^{n-1}} d V_{i K}
$$

Then Eq. (3.20) reads

$$
\int_{S^{n-1}}\left(\frac{h_{L}}{h_{K}}-1\right) d \bar{V}_{i K} \geq \frac{1}{n-i} \log \frac{W_{i}(L)}{W_{i}(K)} .
$$

We call (3.22) the weaker of the $i$ th $\log$ Quermassintegral inequality. In fact,

$$
\frac{h_{L}}{h_{K}}-1 \geq \log \frac{h_{L}}{h_{K}}
$$

for all $u \in S^{n-1}$, and the equality holds if and only if $\frac{h_{L}}{h_{K}}=1$, that is, $K=L$. For $i=0$ and $n=2$, since $d \bar{V}_{0 K}=d \bar{V}_{K}$, the cone volume probability measure of $K$, then by (3.23) and (3.22) we obtain

$$
\int_{S^{1}}\left(\frac{h_{L}}{h_{K}}-1\right) d \bar{V}_{K} \geq \int_{S^{1}} \log \frac{h_{L}}{h_{K}} d \bar{V}_{K} \geq \frac{1}{2} \log \frac{V(L)}{V(K)}
$$

So we have the following corollary. 
Corollary 3.8 Let $K, L \in \mathcal{K}^{n}, W_{i}(K)$ denotes the ith Quermassintegral of $K, \bar{V}_{i K}$ be the normalized $i$-cone volume probability measure. Then

$$
\int_{S^{n-1}}\left(\frac{h_{L}}{h_{K}}-1\right) d \bar{V}_{i K} \geq \frac{1}{n-i} \log \frac{W_{i}(L)}{W_{i}(K)}
$$

When $h_{K}=h_{L}$, equality holds.

\section{Acknowledgements}

The authors would like to strongly thank the anonymous referee for the very valuable comments and helpful suggestions that directly lead to improve the original manuscript.

\section{Funding}

The work is supported in part by CNSF (Grant No. 11561012, 11861024), Guizhou Foundation for Science and Technology (Grant No. [2019] 1055, [2019]1228), Science and technology top talent support program of Guizhou Eduction Department (Grant No. [2017]069).

\section{Availability of data and materials}

Not applicable.

\section{Competing interests}

The authors declare that they have no competing interests.

\section{Authors' contributions}

All authors jointly worked on the results, and they read and approved the final manuscript.

\section{Author details}

${ }^{1}$ School of Mathematics and Statistics, Guizhou University of Finance and Economics, Guiyang, Guizhou, China. ${ }^{2}$ School of Mathematical Sciences, Guizhou Normal University, Guiyang, Guizhou, China.

\section{Publisher's Note}

Springer Nature remains neutral with regard to jurisdictional claims in published maps and institutional affiliations.

Received: 28 September 2020 Accepted: 18 November 2020 Published online: 30 November 2020

\section{References}

1. Alonso-Gutiérrez, D., Merino, B., Jiménez, C., Villa, R.: Rogers-Shephard inequality for log-concave function. J. Funct. Anal. 271, 3269-3299 (2016)

2. Alonso-Gutiérrez, D., Merino, B., Jiménez, C., Villa, R.: John's ellipsoid and the integral ratio of a log-concave function. J. Geom. Anal. 28, 1182-1201 (2018)

3. Artstein-Avidan, S., Milman, V.: A characterization of the support map. Adv. Math. 223(1), 379-391 (2010)

4. Artstein-Avidan, S., Slomka, B.: A note on Santaló inequality for the polarity transform and its reverse. Proc. Am. Math. Soc. 143(4), 1693-1904 (2015)

5. Avidan, S., Klartag, B., Schütt, C., Werner, E.: Functional affine-isoperimetry and an inverse logarithmic Sobolev inequality. J. Funct. Anal. 262, 4181-4204 (2012)

6. Ball, K.: Logarithmically concave functions and sections of convex sets in $\mathbb{R}^{n}$. Stud. Math. 88, 69-84 (1988)

7. Ball, K.: Isometric problems in $I_{p}$ and section of convex sets. Ph.D. dissertation, Cambridge (1986)

8. Barchiesia, F., Böröczky, K., Fradelizi, M.: Stability of the functional forms of the Blaschke-Santaló inequality. Monatshefte Math. 173, 135-159 (2014)

9. Barchiesia, M., Caprianib, G., Fusco, N., Pisante, G.: Stability of Pólya-Szegö inequality for log concave functions. J. Funct. Anal. 267, 2264-2297 (2014)

10. Bobkov, S., Colessanti, A., Fragalà, l.: Quermassintegrals of quasi-concave functions and generalized Prékopa-Leindler inequalities. Manuscr. Math. 143, 131-169 (2014)

11. Böröczky, K., Lutwak, E., Yang, D., Zhang, G.: The log-Brunn-Minkowski inequality. Adv. Math. 231, 1974-1997 (2012)

12. Caglar, U., Werner, E.: Divergence for s-concave and log concave functions. Adv. Math. 257, 219-247 (2014)

13. Caglar, U., Werner, E.: Mixed f-divergence and inequalities for log concave functions. Proc. Lond. Math. Soc. 110(2), 271-290 (2015)

14. Caglar, U., Ye, D.: Affine isoperimetric inequalities in the functional Orlicz-Brunn-Minkowski theory. Adv. Appl. Math. $81,78-114(2016)$

15. Colesanti, A., Fragalà, I.: The first variation of the total mass of log-concave functions and related inequalities. Adv. Math. 244, 708-749 (2013)

16. Fang, N., Zhou, J.: LYZ ellipsoid and Petty projection body for log-concave function. Adv. Math. 340, 914-959 (2018)

17. Fradelizi, M., Gordon, Y., Meyer, M., Reisner, S.: The case of equality for an inverse Santaló inequality. Adv. Geom. 10, 621-630 (2010)

18. Gardner, R.: The Brunn-Minkowski inequality. Bull. Am. Math. Soc. 39, 355-405 (2002)

19. Gardner, R.: Geometric Tomography, 2nd edn. Encyclopedia Math. Appl., vol. 58. Cambridge University Press, Cambridge (2006) 
20. Gutiérrez, D., Avidan, S., Merino, B., Jiménez, C., Villa, R.: Rogers-Shephard and local Loomis-Whitney type inequalities. Math. Ann. 374, 1719-1771 (2019)

21. Haddad, J., Jiménez, C., Montenegro, M.: Asymmetric Blaschke-Santaló functional inequalities. J. Funct. Anal. (2019). https://doi.org/10.1016/j.jfa.2019.108319

22. Klartag, B., Milman, V.: Geometry of log-concave functions and measures. Geom. Dedic. 112, 169-182 (2005)

23. Lin, Y:: Affine Orlicz Pólya-Szegö for log-concave functions. J. Funct. Anal. 273, 3295-3326 (2017)

24. Lutwak, E.:The Brunn-Minkowski-Firey theory I: mixed volumes and the Minkowski problem. J. Differ. Geom. 38 131-150 (1993)

25. Rockafellar, T.: Convex Analysis. Princeton Press, Princeton (1970)

26. Rotem, L:: On the mean width of log-concave functions. In: Geometric Aspects of Functional Analysis, in: Geometric Aspects of Functional Analysis. Lecture Notes in Math., vol. 2050. Springer, Berlin (2012)

27. Rotem, L:: Support functions and mean width for $\alpha$-concave functions. Adv. Math. 243, 168-186 (2013)

28. Rotem, L.: A sharp Blaschke-Santaló inequality for $\alpha$-concave functions. Geom. Dedic. 172, 217-228 (2014)

29. Schneider, R.: Convex Bodies: The Brunn-Minkowski Theory. Encyclopedia Math. Appl., vol. 58. Cambridge University Press, Cambridge (1993)

\section{Submit your manuscript to a SpringerOpen ${ }^{\circ}$ journal and benefit from:}

- Convenient online submission

- Rigorous peer review

Open access: articles freely available online

- High visibility within the field

- Retaining the copyright to your article

Submit your next manuscript at $\gg$ springeropen.com 